\title{
VASCULITIS
}

\section{Epitope specificity responsible for MPO-ANCA pathogenicity}

A new study reports that epitope specificity determines whether or not myeloperoxidase (MPO) autoantibodies are pathogenic, which explains why MPO autoantibodies can be present in individuals without disease.

MPO-specific anti-neutrophil cytoplasmic antibody (ANCA)-associated antibodies are serologic markers that can be used to diagnose some cases of small vessel necrotizing vasculitis, and studies have indicated that ANCAs are crucial in the pathogenesis of ANCA-associated vasculitis (AAV). However, several observations are at odds with the idea that ANCAs are pathogenic: conventional assays do not detect ANCAs in all patients with AAV; ANCA titres do not correlate well with disease activity; and naturally occurring anti-MPO antibodies can occur in healthy individuals.

Roth et al. used highly sensitive epitope excision matrix-assisted laser desorption/ ionization-time of flight (MALDI-TOF)/ TOF-mass spectrometry to determine

\section{These studies explain why asymptomatic or natural MPO autoantibodies can exist in individuals free of disease... 77}

specific epitopes on MPO autoantibodies to try and explain the inconsistencies.

The reseachers found that MPO autoantibodies from patients with AAV had different epitope specificities to MPO autoantibodies found in healthy individuals, and that a specific linear epitope on MPO was solely associated with active disease. Patients with ANCAnegative disease had MPO-ANCAs specific for this epitope, and levels of reactivity towards this peptide correlated with disease activity. A protein fragment in sera was found to interfere with conventional testing in ANCA-negative disease. Autoantibodies against the epitope were also shown to activate neutrophils in vitro and induce nephritis in mice.
"Fundamental questions problematic to the understanding of ANCA vasculitis were resolved, at least in part, by the studies presented here," state the authors. "These studies explain why asymptomatic or natural MPO autoantibodies can exist in individuals free of disease: epitope specificity defines pathogenicity. They explain why there is a lack of correlation between MPO-ANCA titers and active disease: clinical tests do not discriminate between natural anti-MPO autoantibodies and potentially pathogenic ANCA. They explain why some patients with vasculitis are seronegative for ANCA in clinical tests: detection of a monoclonal MPO-ANCA reactive with a small, restricted epitope is obstructed by a protein present in sera."

Rebecca Kelsey

Original article Roth, A. J. et al. Epitope specificity determines pathogenicity and detectability in ANCAassociated vasculitis. J. Clin. Invest. doi:10.1172/ JCl65292 